\title{
On conjectures of Mathai and Borel
}

\author{
Stanley Chang ${ }^{1}$
}

\begin{abstract}
Mathai $[\mathrm{M}]$ has conjectured that the Cheeger-Gromov invariant $\rho_{(2)}=\eta_{(2)}-\eta$ is a homotopy invariant of closed manifolds with torsion-free fundamental group. In this paper we prove this statement for closed manifolds $M$ when the rational Borel conjecture is known for $\Gamma=\pi_{1}(M)$, i.e. the assembly map $\alpha: H_{*}(B \Gamma, \mathbb{Q}) \rightarrow L_{*}(\Gamma) \otimes \mathbb{Q}$ is an isomorphism. Our discussion evokes the theory of intersection homology and results related to the higher signature problem.
\end{abstract}

Let $M$ be a closed, oriented Riemannian manifold of dimension $4 k-1$, with $k \geq 2$. In [Ma] Mathai proves that the Cheeger-Gromov invariant $\rho_{(2)} \equiv \eta_{(2)}-\eta$ is a homotopy invariant of $M$ if $\Gamma=\pi_{1}(M)$ is a Bieberbach group. In the same work, he conjectures that $\rho_{(2)}$ will be a homotopy invariant for all such manifolds $M$ whose fundamental group $\Gamma$ is torsion-free and discrete. This conjecture is verified by Keswani $[\mathrm{K}]$ when $\Gamma$ is torsion-free and the Baum-Connes assembly map $\mu_{\max }: K_{0}(B \Gamma) \rightarrow K_{0}\left(C^{*} \Gamma\right)$ is an isomorphism. Yet it is now known that $\mu_{\max }$ fails to be an isomorphism for groups satisfying Kazhdan's property $T$. This paper improves on Keswani's result by showing that Mathai's conjecture holds for torsion-free groups satisfying the rational Borel conjecture, for which no counterexamples have been found.

As a consequence of a theorem of Hausmann $[\mathrm{H}]$, for every compact odd-dimensional oriented manifold $M$ with fundamental group $\Gamma$, there is a manifold $W$ with boundary such that $\Gamma$ injects into $G=\pi_{1}(W)$ and $\partial W=r M$ for some multiple $r M$ of $M$. Using this result, the author and Weinberger construct in [CW] a well-defined Hirzebruch-type invariant for $M^{4 k-1}$ given by

$$
\tau_{(2)}(M)=\frac{1}{r}\left(\operatorname{sig}_{(2)}^{G}(\widetilde{W})-\operatorname{sig}(W)\right),
$$

where $\widetilde{W}$ is the universal cover of $W$. The $\operatorname{map} \operatorname{sig}_{(2)}^{G}$ is a real-valued homomorphism on the $L$-theory group $L_{4 k}(G)$ given by $\operatorname{sig}_{(2)}^{G}(V)=\operatorname{dim}_{G}\left(V^{+}\right)-\operatorname{dim}_{G}\left(V^{-}\right)$for any quadratic form $V$, considered as an $\ell^{2}(G)$-module. This invariant $\tau_{(2)}$ is in general a diffeomorphism invariant, but is not a homotopy invariant when $\pi_{1}(M)$ is not torsion-free [CW]. It is now also known that $\tau_{(2)}$ coincides with $\rho_{(2)}$ by the work of Lück and Schick [LS].

The purpose of this paper is to show that the diffeomorphism invariant $\tau_{(2)}$, and subsequently $\rho_{(2)}$, is actually a homotopy invariant of $M^{4 k-1}$ if the fundamental group $\Gamma=\pi_{1}(M)$ satisfies the rational Borel conjecture, which states that the assembly map $\alpha: H_{*}(B \Gamma, \mathbb{Q}) \rightarrow L_{*}(\Gamma) \otimes \mathbb{Q}$ is an isomorphism if $\Gamma$ is torsion-free. We include in our discussion some background in $L$-theory and intersection homology.

I would like to thank Shmuel Weinberger for some useful conversations.

\footnotetext{
${ }^{1}$ Research partially supported by NSF Grant DMS-9971657.
} 


\section{Introduction}

The classical work on cobordism by Thom implies that every compact odd-dimensional oriented manifold $M$ has a multiple $r M$ which is the boundary of an oriented manifold $W$. Hausmann $[\mathrm{H}]$ showed furthermore that, for every such $M$ with fundamental group $\Gamma$, there is a manifold $W$ such that $\partial W=r M$, for some multiple $r M$ of $M$, with the additional property that the inclusion $M \hookrightarrow W$ induces an injection $\Gamma \hookrightarrow \pi_{1}(W)$. For such a manifold $M^{4 k-1}$ with fundamental group $\Gamma$, a higher "Hirzebruch type" real-valued function $\tau_{(2)}$ is defined in $[\mathrm{CW}]$ in the following manner:

$$
\tau_{(2)}^{G}(M)=\frac{1}{r}\left(\operatorname{sig}_{(2)}^{G}(\widetilde{W})-\operatorname{sig}(W)\right),
$$

where $G=\pi_{1}(W)$ and $\widetilde{W}$ is the universal cover of $W$.

To see that this quantity can be made independent of $(W, G)$, we consider any injection $G \hookrightarrow G^{\prime}$. Let $W_{G^{\prime}}$ be the $G^{\prime}$-space induced from the $G$-action on $\widetilde{W}$ to $G^{\prime}$. Now define

$$
\tau_{(2)}^{G^{\prime}}(M)=\frac{1}{r}\left(\operatorname{sig}_{(2)}^{G^{\prime}}\left(W_{G^{\prime}}\right)-\operatorname{sig}\left(W_{G^{\prime}} / G^{\prime}\right)\right)
$$

Since $\widetilde{W}$ is simply the $G$-cover $W_{G}$ of $W$ and the quotient $W_{G^{\prime}} / G^{\prime}$ is clearly diffeomorphic to $W$, we note that this definition of $\tau_{(2)}^{G^{\prime}}$ is consistent with the above definition of $\tau_{(2)}^{G}$. However, by the $\Gamma$-induction property of Cheeger-Gromov [CG, page 8, equation (2.3)], we have

$$
\begin{aligned}
\tau_{(2)}^{G^{\prime}}(M) & =\frac{1}{r}\left(\operatorname{sig}_{(2)}^{G^{\prime}}\left(W_{G^{\prime}}\right)-\operatorname{sig}\left(W_{G^{\prime}} / G^{\prime}\right)\right) \\
& =\frac{1}{r}\left(\operatorname{sig}_{(2)}^{G}\left(W_{G}\right)-\operatorname{sig}(W)\right) \\
& =\tau_{(2)}^{G}(M) .
\end{aligned}
$$

So from $G$ one can pass to any larger group $G^{\prime}$ without changing the value of this quantity. Given two manifolds $W$ and $W^{\prime}$ with the required bounding properties, we can use the larger group $G^{\prime}=\pi_{1}(W) *_{\Gamma} \pi_{1}\left(W^{\prime}\right)$, which contains both fundamental groups $\pi_{1}(W)$ and $\pi_{1}\left(W^{\prime}\right)$. The usual Novikov additivity argument ${ }^{2}$ proves that $\tau_{(2)}^{G}(M)$ is independent of all choices $[\mathrm{CW}]$. We will henceforth refer to it as $\tau_{(2)}$.

In $\left[\mathrm{CW}\right.$ ] we prove that $\tau_{(2)}$ is a differential invariant but not a homotopy invariant of $M^{4 k-1}$ when $\pi_{1}(M)$ is not torsion-free. We will use ideas given by Weinberger in [W] which under the same conditions proves the homotopy invariance of the twisted $\rho$-invariant $\rho_{\alpha}(M)=\eta_{\alpha}(M)-$ $\eta(M)$, where $\alpha$ is any representation $\pi_{1}(M) \rightarrow U(n)$. We supply brief expository remarks about intersection homology and algebraic Poincaré complexes in Sections I and II before proving the theorem in Section III.

\footnotetext{
${ }^{2}$ Novikov additivity, proved cohomologically in [AS], posits that signature is additive in the following sense: if $Y$ is an oriented manifold of dimension $2 n$ with boundary $X$ and $Y^{\prime}$ is another such manifold with boundary $-X$, then $\operatorname{sig}\left(Y \cup_{X} Y^{\prime}\right)=\operatorname{sig}(Y)+\operatorname{sig}\left(Y^{\prime}\right)$. The additivity for $\operatorname{sig}_{(2)}^{G}$ is easy to argue on the level of $\ell^{2}(G)$-modules $V$ endowed with nonsingular bilinear form. To see that this additivity corresponds to appropriate manifold glueings, we refer the reader to [F1] and [F2] of Farber, who puts $L^{2}$ cohomology groups into a suitable framework in which the same arguments can be repeated.
} 


\section{Intersection homology}

Following Goresky and MacPherson, we say that a compact space $X$ is a pseudomanifold of dimension $n$ if there is a compact subspace $\Sigma$ with $\operatorname{dim}(\Sigma) \leq n-2$ such that $X-\Sigma$ is an $n$-dimensional oriented manifold which is dense in $X$. We assume that our pseudomanifolds come equipped with a fixed stratification by closed subspaces

$$
X=X_{n} \supset X_{n-1}=X_{n-2}=\Sigma \supset X_{n-3} \supset \cdots \supset X_{1} \supset X_{0}
$$

satisfying various neighborhood conditions (see $[\mathrm{GM}]$ ). A perversity is a sequence of integers $\bar{p}=\left(p_{2}, p_{3}, \cdots, p_{n}\right)$ such that $p_{2}=0$ and $p_{k+1}=p_{k}$ or $p_{k}+1$. A subspace $Y \subset X$ is said to be $(\bar{p}, i)$-allowable if $\operatorname{dim}(Y) \leq i$ and $\operatorname{dim}\left(Y \cap X_{n-k}\right) \leq i-k+p_{k}$. We denote by $I C_{i}^{\bar{p}}$ the subgroup of $i$-chains $\xi \in C_{i}(X)$ for which $|\xi|$ is $(\bar{p}, i)$-allowable and $|\partial \xi|$ is $(\bar{p}, i-1)$-allowable. If $X$ is a pseudomanifold of dimension $n$, then we define the $i$-th intersection homology group $\operatorname{IH}_{i}^{\bar{p}}(X)$ to be the $i$-th homology group of the chain complex $I C_{*}^{\bar{p}}(X)$. For any perversity $\bar{p}$, we have $I H_{0}^{\bar{p}}(X) \cong H^{n}(X)$ and $I H_{n}^{\bar{p}}(X) \cong H_{n}(X)$.

Whenever $\bar{p}+\bar{q} \leq \bar{r}$, the intersection homology groups can be equipped with a unique product $\cap: I H_{i}^{\bar{p}}(X) \times I H_{j}^{\bar{q}}(X) \rightarrow I H_{i+j-n}^{\bar{r}}(X)$ that respects the intersection homology classes of dimensionally transverse pairs $(C, D)$ of cycles. Let $\bar{m}=(0,0,1,1,2,2, \ldots, 2 k-2,2 k-2,2 k-1)$ be the middle perversity. If $X$ is stratified with only strata of even codimension, and if $\operatorname{dim}(X)=$ $4 k$, then the intersection pairing

$$
\cap: I H_{2 k}^{\bar{m}}(X) \times I H_{2 k}^{\bar{m}}(X) \rightarrow \mathbb{Z}
$$

is a symmetric and nonsingular form when tensored with $\mathbb{Q}$. We can define the signature $\operatorname{sig}(X)$ of $X$ to be the signature of this quadratic form. If $X$ is a manifold, this definition coincides with the usual notion of signature.

If $X$ is a pseudomanifold, we say that $X$ is a Witt space if $I H_{k}^{\bar{m}}(L, \mathbb{Q})=0$ whenever $L^{2 k}$ is the link of an odd-codimensional stratum of $X$. If $X^{q}$ is a Witt space, there is a nondegenerate rational pairing

$$
I H_{i}^{\bar{m}}(X, \mathbb{Q}) \times I H_{j}^{\bar{m}}(X, \mathbb{Q}) \rightarrow \mathbb{Q}
$$

whenever $i+j=q$. If $q=4 k>0$, then $I H_{2 k}^{\bar{m}}(X, \mathbb{Q})$ is a symmetric inner product space. In this case, we define the Witt class $w(X)$ of $X$ to be the equivalence class of $I H_{2 k}^{\bar{m}}(X, \mathbb{Q})$ in the Witt ring $W(\mathbb{Q})$ of classes of symmetric rational inner product spaces. If $q=0$, set $w(X)$ to be $\operatorname{rank}\left(H_{0}(X, \mathbb{Q})\right) \cdot\langle 1\rangle$, and set it to zero if $q \not \equiv 0 \bmod 4$. If $(X, \partial X)$ is a Witt space with boundary, set $w(X)=w(\widehat{X})$, where $\widehat{X}=X \cup \operatorname{cone}(\partial X)$. Let $\operatorname{sig}_{\mathbb{Q}}: W(\mathbb{Q}) \rightarrow \mathbb{Z}$ be the signature homomorphism developed by Milnor and Husemoller $[\mathrm{MH}]$. We define the signature $\operatorname{sig}(X)$ to the integer $\operatorname{sig}_{\mathbb{Q}}(w(X))$. See $[\mathrm{S}]$.

Denote by $\Omega_{*}^{\text {Witt }}$ the bordism theory based on Witt spaces; i.e. if $Y$ is a Witt space, define $\Omega_{n}^{\text {Witt }}(Y)$ to be the classes $[X, f]$, where $X$ is an $n$-dimensional Witt space and $f: X \rightarrow Y$ a continuous map, such that $\left[X_{1}, f_{1}\right] \sim\left[X_{2}, f_{2}\right]$ iff there is an $(n+1)$-dimensional Witt space $W$ with $\partial W=X_{1} \amalg X_{2}$ and a map $W \rightarrow Y$ that restricts to $f_{1}$ and $f_{2}$ on the boundary. Witt bordism enjoys the important properties that (1) there is a signature invariant defined on the 
cycle level which is a cobordism invariant, and (2) the signature can be extended to relative cycles $(X, \partial X)$ so that it is additive.

Lemma 1: Let $M_{1}$ and $M_{2}$ be homotopy equivalent manifolds of the same dimension. Suppose that their fundamental group $\Gamma$ is torsion-free and satisfies the rational Borel conjecture. Then there is rationally a Witt cobordism between $M$ and $M^{\prime}$ over $B \Gamma$.

Proof: It is well-known that, if $\Gamma$ is torsion-free and satisfies the Borel conjecture, then it satisfies the Novikov conjecture, which asserts that, if $f: M \rightarrow B \Gamma$ is a map, then the generalized Pontrjagin number (or "higher signature")

$$
f_{*}(L(M) \cap[M]) \in H_{*}(B \Gamma, \mathbb{Q})
$$

is an oriented homotopy invariant. Here $L(M)$ is the Hirzebruch $L$-polynomial in terms of the Pontrjagin classes, and $[M]$ is the fundamental class of $M$. Let $f_{i}: M_{i} \rightarrow B \Gamma$ be the map classifying the universal cover of $M_{i}$. We would like to show that $\left[M_{1}, f_{1}\right]$ and $\left[M_{2}, f_{2}\right]$ rationally define the same class in $\Omega_{*}^{\text {Witt }}(B \Gamma)$.

For a Witt space $X$, Siegel $[\mathrm{S}]$ constructs an $L$-class $L(X) \in H_{*}(X, \mathbb{Q})$ that generalizes the $L$-class of Goresky and MacPherson [GM], the latter of which is defined only on Whitney stratified pseudomanifolds with even-codimensional strata. If $f: X \rightarrow B \Gamma$ is the universal cover of $X$, then $f_{*}(L(X)) \in H_{*}(B \Gamma, \mathbb{Q})$ coincides with the higher signature given above. In addition, it is a bordism invariant in $\Omega_{*}^{\text {Witt }}(B \Gamma)$. Rationally the Atiyah-Hirzebruch spectral sequence for Witt cobordism collapses, so we obtain an isomorphism

$$
i: \Omega_{n}^{\mathrm{Witt}}(B \Gamma) \otimes \mathbb{Q} \longrightarrow \bigoplus_{k \geq 0} H_{n-4 k}(B \Gamma, \mathbb{Q})
$$

such that $i([M, f])$ is the higher signature $f_{*}(L(M) \cap[M])$. But these signatures are homotopy invariants by assumption.

\section{Algebraic Poincaré complexes}

An $n$-dimensional Poincaré complex over a ring $A$ with involution is an $A$-module chain complex $C$ with a collection of $A$-module morphisms $\phi_{s}: C^{n-r+s} \rightarrow C_{r}$ such that the chain map $\phi_{0}$ is a chain equivalence inducing abstract Poincaré duality isomorphisms $\phi_{0}: H^{n-r}(C) \rightarrow H_{r}(C)$. As defined by Wall [Wa], an $n$-dimensional geometric Poincaré complex $X$ with fundamental group $\Gamma$ is a finitely dominated $\mathrm{CW}$-complex together with a fundamental class $[X] \in H_{n}(\widetilde{X}, \mathbb{Z})$ such that cap product with $[X]$ gives a family of $\mathbb{Z} \Gamma$-module isomorphisms

$$
\cap[X]: H^{r}(\widetilde{X}) \longrightarrow H_{n-r}(\widetilde{X})
$$

for $0 \leq r \leq n$ (there is additional business about an orientation group morphism $w: \Gamma_{1}(X) \rightarrow$ $\mathbb{Z}_{2}$ for which one should consult [Ran2]). An $n$-dimensional geometric Poincaré complex $X$ with fundamental group $\Gamma$ naturally determines an $n$-dimensional symmetric Poincaré complex $\left(C(\widetilde{X}), \phi_{\tilde{X}}\right)$ over $\mathbb{Z} \Gamma$. Such symmetric complexes (as opposed to quadratic complexes, which 
define lower $L$-theory) over a ring $A$ can be assembled into an abelian group $L^{n}(A)$ under a cobordism relation defined by abstract Poincaré-Lefschetz duality [M,Ran1], with addition given by

$$
(C, \phi)+\left(C^{\prime}, \phi^{\prime}\right)=\left(C \oplus C^{\prime}, \phi \oplus \phi^{\prime}\right) \in L^{n}(A) .
$$

For a more detailed discussion on algebraic Poincaré complexes and in particular the manner in which algebraic Poincaré complexes are glued together along a common boundary, see [Ran3].

Lemma 2: Let $M$ and $M^{\prime}$ be homotopy equivalent manifolds of the same dimension $4 k-1$ with torsion-free fundamental group $\Gamma$. Suppose that $Y$ is a rational Witt cobordism of $M$ and $M^{\prime}$ over $B \Gamma$. If the Borel conjecture holds for $\Gamma$, then $\operatorname{sig}_{(2)}^{\Gamma}\left(Y_{\Gamma}\right)=\operatorname{sig}(Y)$, where $Y_{\Gamma}$ is the induced $\Gamma$-cover of $Y$.

Proof: By the preceding lemma, there is a Witt cobordism between $n$ copies of $M$ and $n$ copies of $M^{\prime}$. Let $C^{h}$ be the homotopy cylinder given by the homotopy equivalence $h: M \rightarrow M^{\prime}$. Attach $n$ copies of $C^{h}$ to the rational Witt cobordism $Y$ to form a space $X$. This space is usually not a pseudomanifold because the singular space is of codimension one, but it is an algebraic Poincaré space. Notice that all of these spaces come equipped with a map to $B \Gamma$. Since upper and lower $L$-theory coincide rationally, we may then consider $X$ as an element of $L_{*}(B \Gamma) \otimes \mathbb{Q}$. Consider then the composition of maps

$$
\Omega_{*}^{\mathrm{SO}}(B \Gamma) \otimes \mathbb{Q} \longrightarrow H_{*}(B \Gamma, \mathbb{Q}) \longrightarrow L_{*}(\Gamma) \otimes \mathbb{Q} .
$$

The first map is well known to be surjective, and the second is surjective by our assumption that the Borel conjecture holds for $\Gamma$. Therefore there is a closed manifold $X^{\prime}$ without boundary bordant to $X$ as algebraic Poincaré complexes. Atiyah's $\Gamma$-index theorem $[\mathrm{A}]$ shows that $\operatorname{sig}_{(2)}^{\Gamma}\left(X_{\Gamma}^{\prime}\right)=\operatorname{sig}\left(X^{\prime}\right)$, so that the cobordism invariance of signature implies that $\operatorname{sig}_{(2)}^{\Gamma}\left(X_{\Gamma}\right)=\operatorname{sig}(X)$. For a topological proof of the $\Gamma$-index theorem for signature, see the appendix of $[\mathrm{CW}]$.

By Novikov additivity, we may decompose the complex $X$ to arrive at the equation

$$
\operatorname{sig}_{(2)}^{\Gamma}\left(Y_{\Gamma}\right)+n \cdot \operatorname{sig}_{(2)}^{\Gamma}\left(C_{\Gamma}^{h}\right)=\operatorname{sig}(Y)+n \cdot \operatorname{sig}\left(C^{h}\right) .
$$

By Novikov additivity, the homotopy cylinder $C^{h}$ enjoys the convenient property that $\operatorname{sig}_{(2)}^{\Gamma}\left(C_{\Gamma}^{h}\right)=$ $\operatorname{sig}\left(C^{h}\right), \operatorname{so~} \operatorname{sig}_{(2)}^{\Gamma}\left(Y_{\Gamma}\right)=\operatorname{sig}(Y)$.

\section{The main theorem}

Theorem: Let $M$ be an oriented compact manifold of dimension $4 k-1$. If $\pi_{1}(M)$ is torsion-free, then the real-valued quantity $\tau_{(2)}(M)$ is a homotopy invariant.

Proof: Let $M$ and $M^{\prime}$ be homotopy equivalent closed manifolds of dimension $n=4 k-1$. There are closed manifolds $W$ and $W^{\prime}$ such that $\partial W=r M$ and $\partial W^{\prime}=s M^{\prime}$ with injecting fundamental groups. Construct $s$ copies of $W$ and $r$ copies of $W^{\prime}$ and attach $r s$ Witt cobordisms $Y$ between the boundary components $M$ and $M^{\prime}$. Call this space $X$ with $H=\pi_{1}(X)$. Note that both 
$\pi_{1}(W)$ and $\pi_{1}\left(W^{\prime}\right)$ inject into $H$. Since the map $\Omega^{\mathrm{SO}}(B H) \otimes \mathbb{Q} \rightarrow \Omega^{\mathrm{Witt}}(B H) \otimes \mathbb{Q}$ is surjective (see $[\mathrm{Cu}]$ ), it follows that $X$ is Witt cobordant to a smooth manifold $X^{\prime}$. Hence they share the same signatures. Note that $X^{\prime}$ has the property that $\operatorname{sig}_{(2)}^{H}\left(X_{H}^{\prime}\right)=\operatorname{sig}\left(X^{\prime}\right)$, and hence $\operatorname{sig} \Gamma_{(2)}\left(X_{\Gamma}^{\prime}\right)=\operatorname{sig}\left(X^{\prime}\right)$ by Atiyah's $\Gamma$-index theorem. By the observation above, we can then conclude that $\operatorname{sig}_{(2)}^{\Gamma}\left(X_{\Gamma}\right)=\operatorname{sig}(X)$.

By Novikov additivity, we can partition $X$ along the original attachments to obtain

$$
s \cdot \operatorname{sig}_{(2)}^{\Gamma}\left(W_{\Gamma}\right)+r s \cdot \operatorname{sig}_{(2)}^{\Gamma_{(2)}}\left(Y_{\Gamma}\right)-r \cdot \operatorname{sig}_{(2)}^{\Gamma_{(2)}}\left(W_{\Gamma}\right)=s \cdot \operatorname{sig}(W)+r s \cdot \operatorname{sig}(Y)-r \cdot \operatorname{sig}\left(W^{\prime}\right) .
$$

By Lemma 2, the cylindrical terms cancel, leaving

$$
\frac{1}{r}\left(\operatorname{sig}_{(2)}^{\Gamma}\left(W_{\Gamma}\right)-\operatorname{sig}(W)\right)=\frac{1}{s}\left(\operatorname{sig}_{(2)}^{\Gamma_{2}}\left(W_{\Gamma}^{\prime}\right)-\operatorname{sig}\left(W^{\prime}\right)\right) .
$$

Equivalently $\tau_{(2)}(M)=\tau_{(2)}\left(M^{\prime}\right)$, so $\tau_{(2)}$ is a homotopy equivalence.

Remark: Given the identification of $\tau_{(2)}$ and $\rho_{(2)}$, one can conjecture that $\rho_{(2)}$ is homotopy invariant iff the fundamental group of $M$ is torsion-free (see [CW]). However, recent work of $[\mathrm{NW}]$ makes use of secondary invariants for groups with unsolvable word problems, which, a fortiori, are not residually finite. Potentially, the results of this paper can have applications to the geometry of certain moduli spaces.

\section{References}

[A] M. Atiyah, Elliptic operators, discrete groups and von Neumann algebras, Astérisque 32-33 (1976), 43-72.

[AS] M. Atiyah and I. Singer, The index of elliptic operators. III, Ann. of Math. (2) 871968 546-604.

[CW] S. Chang and S. Weinberger, On invariants of Hirzebruch and Cheeger-Gromov. Geometry and Topology, to appear.

[CG] J. Cheeger and M. Gromov, Bounds on the von Neumann dimension of $L^{2}$-cohomology and the Gauss-Bonnet theorem for open manifolds, J. Differential Geom. 21 (1985), 1-34.

[Cu] S. Curran, Intersection homology and free group actions on Witt spaces. Michigan Math. J. 39 (1992), no. $1,111-127$.

[F1] M. Farber, von Neumann categories and extended $L^{2}$-cohomology, K-Theory 15 (1998), no. 4, 347-405.

[F2] M. Farber, Homological algebra of Novikov-Shubin invariants and Morse inequalities, Geom. Funct. Anal. 6 (1996), no. 4, 628-665.

[GM] M. Goresky and R. MacPherson, Intersection homology theory, Topology 19 (1980), no. 2, $135-162$.

[H] J.-C. Hausmann, On the homotopy of nonnilpotent spaces, Math. Z. 178 (1981), no. 1, 115-123.

[K] N. Keswani, Von Neumann eta-invariants and $C^{*}$-algebra K-theory, J. London Math. Soc. (2) 62 (2000), no. $3,771-783$. 
[LS] W. Lück and T. Schick, Various $L^{2}$-signatures and a topological $L^{2}$-signature theorem, preprint.

[Ma] V. Mathai, Spectral flow, eta invariants and von Neumann algebras, Journal of Functional Analysis 109 (2), 1992.

[MH] J. Milnor and D. Husemoller, Symmetric bilinear forms, Ergebnisse der Mathematik und ihrer Grenzgebiete, Band 73. Springer-Verlag, New York, 1973.

[M] A.S. Mishchenko, Homotopy invariants of non-simply-connected manifolds III. Higher signatures. Izv. Akad. Nauk SSSR ser. mat. 35 (1971), 1316-135.

[NW] A. Nabutovsky and S. Weinberger, The fractal geometry of Riem/Diff, Geometriae Dedicata (to appear).

[Ram] M. Ramachandran, von Neumann index theorems for manifolds with boundary. J. Differential Geom. 38 (1993), no. 2, 315-349.

[Ran1] A. Ranicki, The algebraic theory of surgery. I. Foundations. Proc. London Math. Soc. (3) 40 (1980), no. 1, 87-192.

[Ran2] A. Ranicki, The algebraic theory of surgery. II. Applications to topology. Proc. London Math. Soc. (3) 40 (1980), no. 2, 193-283.

[Ran3] A. Ranicki, An introduction to algebraic surgery, Surveys on surgery theory, Vol. 2, 81-163, Ann. of Math. Stud., 149, Princeton Univ. Press, Princeton, NJ, 2001.

[S] P.H. Siegel, Witt spaces: a geometric cycle theory for KO-homology at odd primes, Amer. J. Math. 105 (1983), no. 5, 1067-1105.

[Wa] C.T.C. Wall, Poincaré complexes. I. Ann. of Math. (2) 861967 213-245.

[W] S. Weinberger, Homotopy invariance of $\eta$-invariants. Proc. Nat. Acad. Sci. U.S.A. 85 (1988), no. 15, 5362-5363.

106 Central Street, Wellesley College, Wellesley MA 02481 